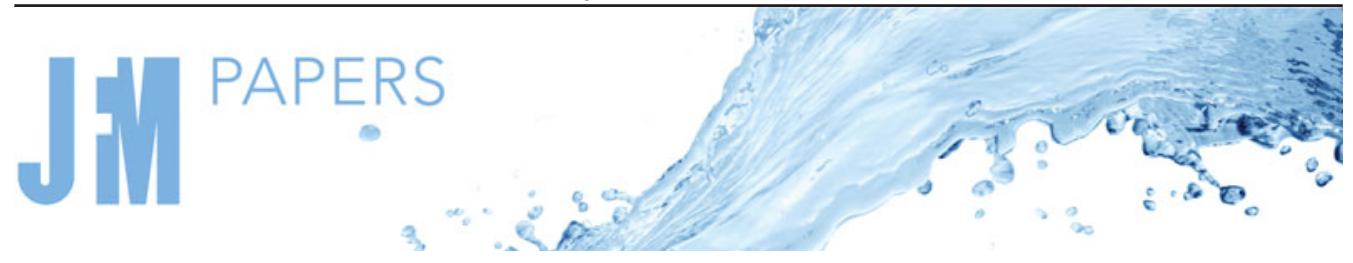

\title{
Mean velocity and temperature profiles in turbulent Rayleigh-Bénard convection at low Prandtl numbers
}

\author{
Wei Xu ${ }^{1}$, Yin Wang ${ }^{2}$, Xiaozhou $\mathrm{He}^{3}$, Xiaoping Wang ${ }^{4}$, Jörg Schumacher ${ }^{5}$, \\ Shi-Di Huang ${ }^{6}$ and Penger Tong ${ }^{2, \dagger}$ \\ ${ }^{1}$ Nano Science and Technology Program, Hong Kong University of Science and Technology, Clear Water \\ Bay, Kowloon, Hong Kong \\ ${ }^{2}$ Department of Physics, Hong Kong University of Science and Technology, Clear Water Bay, Kowloon, \\ Hong Kong \\ ${ }^{3}$ School of Mechanical Engineering and Automation, Harbin Institute of Technology, Shenzhen, PR China \\ ${ }^{4}$ Department of Mathematics, Hong Kong University of Science and Technology, Clear Water Bay, \\ Kowloon, Hong Kong \\ ${ }^{5}$ Institut für Thermo- und Fluiddynamik, Postfach 100565, Technische Universität Ilmenau, D-98684 \\ Ilmenau, Germany \\ ${ }^{6}$ Center for Complex Flows and Soft Matter Research and Department of Mechanics and Aerospace \\ Engineering, Southern University of Science and Technology, Shenzhen 518055, PR China
}

(Received 26 November 2020; revised 27 February 2021; accepted 16 March 2021)

We report a direct numerical simulation (DNS) study of the mean velocity and temperature profiles in turbulent Rayleigh-Bénard convection (RBC) at low Prandtl numbers $(\mathrm{Pr})$. The numerical study is conducted in a vertical thin disk with $\operatorname{Pr}$ varied in the range $0.17 \leq$ $P r \leq 4.4$ and the Rayleigh number $(R a)$ varied in the range $5 \times 10^{8} \leq R a \leq 1 \times 10^{10}$. By varying $\operatorname{Pr}$ from 4.4 to 0.17 , we find a sharp change of flow patterns for the large-scale circulation (LSC) from a rigid-body rotation to a near-wall turbulent jet. We numerically examine the mean velocity equation in the bulk region and find that the mean horizontal velocity profile $u(z)$ can be determined by a balance equation between the mean convection and turbulent diffusion with a constant turbulent viscosity $v_{t}$. This balance equation admits a self-similarity jet solution, which fits the DNS data well. In the boundary-layer region, we find that both the mean temperature profile $T(z)$ and $u(z)$ can be determined by a balance equation between the molecular diffusion and turbulent diffusion. Within the viscous boundary layer, both $u(z)$ and $T(z)$ can be solved analytically and the analytical results agree well with the DNS data. Our careful characterisation of the mean velocity

$†$ Email address for correspondence: penger@ust.hk

(C) The Author(s), 2021. Published by Cambridge University Press. This is an Open Access article, distributed under the terms of the Creative Commons Attribution licence (http://creativecommons.org/ licenses/by/4.0/), which permits unrestricted re-use, distribution, and reproduction in any medium, provided the original work is properly cited. 


\section{W. Xu and others}

and temperature profiles in low- $\operatorname{Pr} \mathrm{RBC}$ provides a further understanding of the intricate interplay between the LSC, plume emission and boundary-layer dynamics, and pinpoints the physical mechanism for the emergence of a pronounced LSC in low- $P r$ RBC.

Key words: Bénard convection, buoyant boundary layers, turbulent convection

\section{Introduction}

Thermal convection at low Prandtl numbers is a common phenomenon both in nature and in many engineering applications. Here, the Prandtl number is defined as $\operatorname{Pr}=v / \kappa$, it represents the relative importance of the kinematic viscosity $v$ to the thermal diffusivity $\kappa$ of the convecting fluid. For example, astronomical observations showed that the Sun has an outer convecting layer of low-density plasma, where the value of $\mathrm{Pr}$ is in the range of $10^{-7}-10^{-4}$ (Hanasoge, Gizon \& Sreenivasan 2016; Schumacher \& Sreenivasan 2020). Seismology measurements showed that the outer core of the Earth is a fluid layer made of a liquid iron-nickel alloy with $\operatorname{Pr} \sim 10^{-2}$ (Stevenson 1981). The convection of the Earth's outer core is linked to the generation and reversal of the Earth's magnetic field (Glatzmaier \& Roberts 1995). The convection of the Earth's atmosphere with $P r \simeq 0.7$ drives the large-scale atmospheric circulation and plays a critical role in the global climate and water balance (Hartmann, Moy \& Fu 2001). Liquid-metal convection is also used in material processing (Brodova, Popel \& Eskin 2001), nuclear engineering (Ihli et al. 2008), and liquid-metal batteries for renewable energy storage (Wang et al. 2014).

In the laboratory, controlled Rayleigh-Bénard convection (RBC) experiments are conducted in a closed cell, in which a fluid layer is heated from below and cooled from above. In addition to the Prandtl number $P r$, the Rayleigh number $R a$ is another control parameter of thermal convection, which is defined as $R a=g \alpha \Delta T H^{3} /(\nu \kappa)$, where $g$ is the gravitational acceleration, $\alpha$ is the thermal expansion coefficient of the fluid and $\Delta T$ is the temperature difference across the fluid layer of height $H$. When the Rayleigh number is sufficiently large (e.g. $R a \gtrsim 10^{8}$ for $P r \simeq 4.4$ ), the bulk fluid becomes turbulent and a large-scale circulation (LSC) is formed across the convection cell (Krishnamurti \& Howard 1981; Zocchi, Moses \& Libchaber 1990). The LSC is driven by the warm and cold plumes emitted from the unstable thermal boundary layers near the bottom and top conducting plates and is maintained in a turbulent environment. This large-scale flow with $\operatorname{Pr}>1$ has been studied extensively in upright cylindrical cells of aspect ratio unity (Du \& Tong 2000; Qiu \& Tong 2001; Xi, Lam \& Xia 2004; Sun, Xia \& Tong 2005), in which the LSC has a single roll structure, with its size comparable to the cell height.

Another intriguing feature of turbulent $\mathrm{RBC}$ is that its thermal boundary layer has significant fluctuations resulting from intermittent eruption of thermal plumes from the boundary layer, even when the boundary layer is not fully turbulent (Du \& Tong 2000; du Puits, Resagk \& Thess 2010; Zhou \& Xia 2010). The structure and dynamics of the thermal boundary layer and its interaction with the LSC are of great importance in determining the global heat transport of the system (Kadanoff 2001; Ahlers, Grossmann \& Lohse 2009). For these reasons, the past decade has witnessed a continuing growth of experimental and theoretical efforts aimed at understanding the boundary-layer dynamics in turbulent RBC. Recent studies of the thermal boundary layer for $\operatorname{Ra} \lesssim 10^{12}$ and $\operatorname{Pr} \gtrsim 1$ (Belmonte, Tilgner \& Libchaber 1993, 1994; Lui \& Xia 1998; Du \& Tong 2000; van Reeuwijk, Jonker \& Hanjalić 2008; Scheel, Kim \& White 2012; Shi, Emran \& Schumacher 2012; Stevens et al. 2012; Wagner, Shishkina \& Wagner 2012; van der Poel, Stevens 


\section{Mean velocity and temperature profiles at low Prandtl numbers}

\& Lohse 2013; du Puits, Resagk \& Thess 2013; Shishkina, Horn \& Wagner 2013; Zhou \& Xia 2013; Scheel \& Schumacher 2014) showed that the measured (and numerically calculated) normalised mean temperature profile $\theta(z)$ has a universal form $\theta(\xi)$, where $\xi \equiv z / \delta_{T}$ is the vertical distance from the conducting plate normalised by the thermal boundary-layer thickness $\delta_{T}$. The measured $\theta(\xi)$ was found to be invariant with $R a$ and has the Prandtl-Blasius-Pohlhausen (PBP) form (Landau \& Lifshitz 1987; Schlichting \& Gersten 2016) for a laminar boundary layer (Grossmann \& Lohse 2000) only when $\xi$ is in the region $\xi \lesssim 0.6$ (Zhou \& Xia 2013). Deviations of $\theta(\xi)$ from the PBP form were found when $0.6 \lesssim \xi \lesssim 4$ (Scheel et al. 2012; Shi et al. 2012; Wagner et al. 2012; du Puits et al. 2013). More recently, Shishkina et al. (2015) considered the effect of boundary-layer fluctuations and obtained an analytical form of $\theta(\xi)$ for the thermal boundary layers with $\operatorname{Pr}>1$, which explained the observed deviations of $\theta(\xi)$ from the PBP form. The theoretical prediction made by Shishkina et al. (2015) was verified by the convection experiments conducted in a quasi-two-dimensional (quasi-2-D) thin-disk cell (Wang, He \& Tong 2016). Wang et al. (2018) further extended the boundary-layer theory to the temperature variance profile.

Compared with the large number of investigations on the dynamics of the LSC and boundary layers for $\operatorname{Pr} \gtrsim 1$, our understanding on the LSC and boundary-layer dynamics for fluids with $\operatorname{Pr}<1$ is rather limited. This is partially caused by the fact that laboratory experiments and numerical simulations of turbulent RBC at low $\operatorname{Pr}$ are quite challenging. On the experimental side, liquid metals, which are often used as a working fluid to obtain a sufficiently low value of $\mathrm{Pr}$, are opaque and thus exclude optical imaging or particle tracking of the velocity field. Owing to their high thermal conductivity, it is also quite difficult to drive the low- $P r$ convection to reach sufficiently high Rayleigh numbers. On the numerical simulation side, because the Kolmogorov scale in the low- $\operatorname{Pr}$ fluid is smaller than the Batchelor scale by a factor of $\operatorname{Pr}^{1 / 2}$ (Grötzbach 1983; Shishkina et al. 2010), a higher spatial resolution is needed to resolve the smaller Kolmogorov scale for low- $\mathrm{Pr}$ convection at a comparable value of $R a$.

Early low- $P r$ experiments using liquid mercury $(P r \simeq 0.024)$ (Takeshita et al. 1996; Cioni, Ciliberto \& Sommeria 1997; Mashiko et al. 2004; Tsuji et al. 2005) or gases (such as nitrogen and sulfur hexafluoride) and gas mixtures $(0.18 \leq P r \leq 0.88)$ (Hogg \& Ahlers 2013) studied the Nusselt number (normalised heat flux) scaling, thermal boundary-layer profile, LSC dynamics and single-point velocity and temperature statistics in the bulk region. Recent low- $P r$ experiments using liquid gallium and its alloys ( $P r \simeq 0.027)$ (Vogt et al. 2018; Zürner et al. 2019) investigated the three-dimensional (3-D) structure and dynamics of LSC in the $R a$ range $10^{5} \lesssim R a \lesssim 6 \times 10^{7}$. These experiments found that the LSC at low $\operatorname{Pr}$ is more coherent compared with that at high $\operatorname{Pr}(>1)$. Recent advances in computational power and numerical techniques allow the study of turbulent RBC at low $\operatorname{Pr}$ by direct numerical simulation (DNS) (Schumacher, Götzfried \& Scheel 2015; Scheel \& Schumacher 2016, 2017; Schumacher et al. 2016). It was found that the mean streamwise velocity is like a near-wall jet (Scheel \& Schumacher 2017). Many of the experimental and DNS studies were conducted in upright cylindrical cells with an aspect ratio close to unity. In the cylindrical cells, the large-scale flow has several 3-D flow modes, such as the torsional and sloshing modes (Funfschilling \& Ahlers 2004; Brown \& Ahlers 2008, 2009; Xi et al. 2009; Ji \& Brown 2020), which may cause additional complications to the study of the LSC and boundary-layer dynamics. There are also corner flows in the closed cylinder (Sun et al. 2005), which may destabilise the large-scale flow (Sugiyama et al. 2010). The strong coupling between the boundary-layer dynamics and complex 3-D large-scale flow in a closed cylinder, which has been studied in recent 


\section{W. Xu and others}

(a)

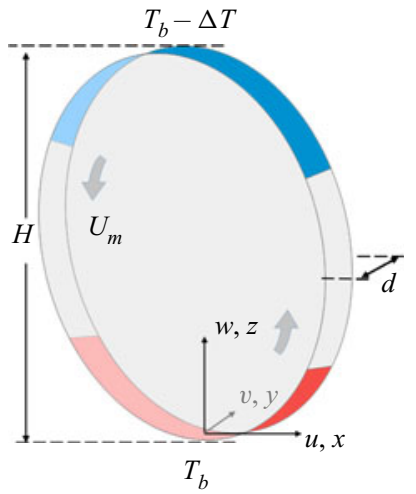

(b)

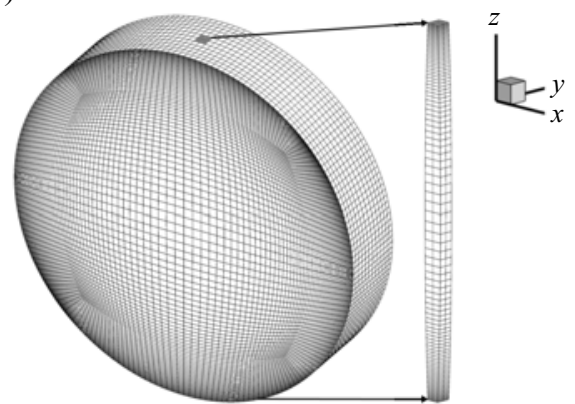

Figure 1. (a) Sketch of the convection cell used for numerical simulations. The convection cell is a vertical thin disk of height $H$ and thickness $d$. The bottom and top 1/3 of the curved sidewall are made of conducting plates whose temperature is kept at constant with $T_{b}$ and $T_{b}-\Delta T$, respectively. All the other walls are thermally insulating. The arrows indicate the direction of the LSC with a maximum velocity $U_{m}$. The local velocity components and spatial coordinates used in the simulation and data analysis are shown at the bottom centre of the convection cell. (b) A thin column surrounding the vertical $z$-axis of the cell is used to compute the time-averaged properties of the flow as a function of $z$. The horizontal cross-section of the thin column has 4 primary elements and 256 nodes, which form a small area of 0.027 thickness square and are used to calculate the time-averaged properties of the flow.

numerical simulations (van Reeuwijk et al. 2008; Scheel et al. 2012; Shi et al. 2012; Stevens et al. 2012; Wagner et al. 2012; van der Poel et al. 2013; Shishkina et al. 2013; Scheel \& Schumacher 2014), makes a quantitative comparison between the experiment and 2-D theory difficult.

In this paper, we report a systematic numerical study of the mean horizontal velocity profile and mean temperature profile for turbulent RBC in the low- $P r$ regime. DNS runs were performed in a vertical thin disk with its circular cross-section aligned parallel to gravity (see figure 1 for details). This specially designed quasi-2-D convection cell has two unique features for the DNS study attempted here. First, because the cell shape matches the single-roll structure of the LSC perfectly, there is no corner flow inside the cell. The LSC in the circular cross-section has a steady rotation along a fixed orientation. Owing to the axial symmetry of the thin disk cell, the initial orientation of the LSC is random for different initial conditions and different values of $R a$. Second, because the flow is confined in a thin circular disk, no 3-D flow modes can be excited in this system. The quasi-2-D flow in the thin disk cell, therefore, has a better geometry satisfying the assumption of the boundary-layer theory for a 2-D flow. Even with these simplifications, the quasi-2-D system retains the key features of turbulent convection, which have been observed in the upright cylinders, and has been used in recent experimental and numerical studies of the LSC dynamics (Song \& Tong 2010; Song, Villermaux \& Tong 2011; Song et al. 2014) and thermal boundary-layer profiles with $\operatorname{Pr}>1$ (Wang et al. 2016, 2018). This is a 'simple but not simpler' convection system, which offers a natural platform to study the dynamics of the LSC and boundary layers, and their interactions in the low-Pr regime, and to test different theoretical ideas.

In this DNS study, we vary the Rayleigh number in the range $5 \times 10^{8} \leq R a \leq 1 \times 10^{10}$ and focus our attention on $\operatorname{Pr}=0.17$. This is the lowest value of $\operatorname{Pr}$ that can be reached by using a gas mixture of $\mathrm{H}_{2}$ and Xe (Bajaj, Ahlers \& Pesch 2002), and we choose this value of $\operatorname{Pr}$ so that the DNS results can be used for comparison with future experiment. This value of $\mathrm{Pr}$ is small enough so that the viscous and thermal boundary layers are well separated 
with the viscous boundary layer being nested within the thermal boundary layer. In this case, the LSC can produce a strong shear to the thermal boundary layer, which suppresses the emission of thermal plumes from the thermal boundary layer. On the other hand, the value of $\mathrm{Pr}$ used is not too small so that we can carry out the DNS study at high Rayleigh numbers with an adequate spatial resolution using the available computational resources.

The remainder of the paper is organised as follows. We first present the numerical method used and DNS set-up in $\S 2$. A comparison of the temperature and velocity fields between the low- $P r$ and high- $P r$ regimes is given in $\S 3$. The DNS results of the mean horizontal velocity and temperature profiles in the boundary-layer region are presented in $\S 4$. The DNS results of the mean horizontal velocity profile in the bulk region are presented in $\S 5$. Finally, the findings of this study are summarised in $\S 6$.

\section{Direct numerical simulation}

The governing equations for turbulent RBC are the incompressible Navier-Stokes equations and the convective heat equation under the Boussinesq approximation. The dimensionless forms of these equations are given by

$$
\begin{gathered}
\hat{\nabla} \cdot \hat{\boldsymbol{u}}=0, \\
\hat{\boldsymbol{u}}_{\hat{t}}+(\hat{\boldsymbol{u}} \cdot \hat{\nabla}) \hat{\boldsymbol{u}}=-\hat{\nabla} \hat{p}+\frac{1}{\sqrt{\operatorname{Ra} / P r}} \hat{\nabla}^{2} \hat{\boldsymbol{u}}+\hat{T} \boldsymbol{e}_{\hat{z}} \\
\hat{T}_{\hat{t}}+(\hat{\boldsymbol{u}} \cdot \hat{\nabla}) \hat{T}=\frac{1}{\sqrt{\operatorname{RaPr}}} \hat{\nabla}^{2} \hat{T} .
\end{gathered}
$$

The length, time, velocity, pressure and temperature are made dimensionless by the cell height $H$, the free-fall time $T_{f}=\sqrt{H /(g \alpha \Delta T)}$, the free-fall velocity $U_{f}=\sqrt{g \alpha \Delta T H}$, the free-fall pressure $p_{f}=\rho g \alpha \Delta T H$ and the temperature difference $\Delta T$ across the cell, respectively.

Figure 1 $(a)$ shows a sketch of the convection cell used for numerical simulations. The dimensionless boundary conditions are given by

$$
\begin{gathered}
\left.\hat{\boldsymbol{u}}\right|_{\text {wall }}=0, \\
\left.\boldsymbol{n} \cdot \hat{\boldsymbol{\nabla}} \hat{T}\right|_{\text {non-conducting walls }}=0, \\
\left.\hat{T}\right|_{\text {bottom }}=0.5 \\
\left.\hat{T}\right|_{\text {top }}=-0.5
\end{gathered}
$$

Another control parameter is the aspect ratio $\Gamma=d / H$, where $d$ is the thickness of the thin disk cell.

The governing equations are solved numerically using the open-source code Nek5000 (Fischer 1997), which uses a spectral element method to accurately resolve the gradients in the velocity field $\hat{\boldsymbol{u}}(\boldsymbol{r}, t)$ and temperature field $\hat{T}(\boldsymbol{r}, t)$. In the simulation, the time-derivative terms are discretised by backward-differentiation formula, the nonlinear convective terms are treated explicitly and the linear diffusive terms are approximated implicitly. This scheme leads to a Poisson equation for pressure and Helmholtz equations for velocity components and temperature. These equations are written in a weak formulation and discretised by the Galerkin method using the $N$ th-order Lagrangian interpolation polynomials as the basis functions on Gauss-Lobatto-Legendre (GLL) collocation points 
(Deville, Fischer \& Mund 2002). More details of the numerical scheme and mesh resolution requirements can be found in Fischer (1997), Deville et al. (2002) and Scheel, Emran \& Schumacher (2013). All the gradients in the post-processing are also calculated on the GLL collocation points with spectral accuracy.

The computational mesh is designed for the highest value of $R a=1 \times 10^{10}$ with $\operatorname{Pr}=0.17$ and the minimal primary mesh size near the boundary is set at $1.944 \times 10^{-3} \mathrm{H}$, which is closed to the estimated viscous boundary-layer thickness. All the simulations are performed with the polynomial order $N=7$, so that we have $8^{3}=512$ grid points within each primary element. We verify that the mesh resolution at $R a=1 \times 10^{10}$ and $\operatorname{Pr}=0.17$ satisfies the Grötzbach's criterion (Grötzbach 1983; Scheel et al. 2013). We use the same mesh for other simulations at lower values of $R a$ and with $\mathrm{Pr}$ in the range 0.1-4.4. This is done to save the programming time during the post-processing of the DNS results, and at the same time we have a sufficient mesh resolution for all simulations. An adaptive time step is applied to ensure that the Courant number is always below 0.5 during the simulations. The viscous and thermal boundary layers are well resolved by using the polynomial order $N=7$. We run each simulation for at least $100 T_{f}$ to reach the steady state, followed by a continuous running for at least another $200 T_{f}$ to conduct time averaging. As shown in figure $1(b)$, the vertical profile of the local properties is computed along a thin column surrounding the vertical $z$-axis of the cell with $x=y=0$ and is averaged over the cross-section of the thin column with a small area of 0.027 thickness square. Other details about the numerical simulations can be found in Wang et al. (2018).

Table 1 lists a summary of the parameters used for simulations at different Rayleigh numbers and Prandtl numbers. In table 1, we also include the numerically calculated values of the local Nusselt number $\mathrm{Nu}$, the Reynolds number $R e$, the normalised viscous boundary-layer thickness $\delta_{v} / H$ and the normalised thermal boundary-layer thickness $\delta_{T} / H$. Here the local Nusselt number is defined as $N u=-\left.\partial_{z} T\right|_{z=0} H / \Delta T$ and the Reynolds number is defined as $R e=U_{m} H / v$. The definition of the viscous and thermal boundary-layer thicknesses, $\delta_{v}$ and $\delta_{T}$, are given in (4.4) and (4.18), respectively. For all the DNS runs, we use the same aspect ratio $\Gamma=0.2$ and the same total number of spectral elements, $N_{e}=96768$.

\section{Comparison of the temperature and velocity fields between the low- and high-Pr fluids}

Figure 2 shows the contour plots of the instantaneous 2-D temperature field on the middle cross-section for two Prandtl numbers: $\operatorname{Pr}=0.17$ and $\operatorname{Pr}=4.4$. It is found that warm rising plumes (in red) are generated from the bottom conducting plate and cold falling plumes (in blue) are generated from the top plate. These plumes drive the LSC. Because the low- $P r$ fluid has a relatively larger thermal diffusivity, the thermal plumes in the low- $P r$ fluid have a shorter lifetime. As a result, they have a lower chance to move into the bulk region and most of them are concentrated in the narrow region near the curved sidewall. On the other hand, the thermal plumes in the high- $P r$ fluid have a longer lifetime and thus they have a higher chance to be found in the bulk region.

The spatial distribution of the thermal plumes has a profound influence on the velocity field. Figure 3 shows a comparison of the in-plane mean velocity field and corresponding mean pressure field across the middle cross-section of the cell between two Prandtl numbers: (a) $P r=0.17$ and (b) $P r=4$.4. It is seen that the mean flow field in the low- $P r$ fluid is more coherent and has a mean flow concentrated mainly in the narrow region near the circular sidewall. The pressure contour in the bulk region shows a homogeneous 


\begin{tabular}{|c|c|c|c|c|c|c|c|c|}
\hline Run & $R a$ & $\operatorname{Pr}$ & $\mathrm{Nu}$ & $\operatorname{Re}\left(\times 10^{3}\right)$ & $\delta_{v} / H\left(\times 10^{-3}\right)$ & $\delta_{T} / H\left(\times 10^{-3}\right)$ & $\left(N_{B L}\right)_{v}$ & $\left(N_{B L}\right)_{T}$ \\
\hline 1 & $1 \times 10^{9}$ & 4.4 & $65.84 \pm 1.05$ & $1.53 \pm 0.01$ & 13.44 & 7.64 & 44 & 26 \\
\hline 2 & $1 \times 10^{9}$ & 2.0 & $62.88 \pm 0.56$ & $3.78 \pm 0.03$ & 10.00 & 8.04 & 33 & 27 \\
\hline 3 & $1 \times 10^{9}$ & 1.0 & $66.71 \pm 0.68$ & $7.54 \pm 0.02$ & 7.09 & 7.71 & 25 & 26 \\
\hline 4 & $1 \times 10^{9}$ & 0.7 & $66.23 \pm 0.03$ & $10.33 \pm 0.01$ & 6.07 & 7.65 & 21 & 26 \\
\hline 5 & $1 \times 10^{9}$ & 0.17 & $58.99 \pm 0.29$ & $32.66 \pm 0.06$ & 3.51 & 8.94 & 12 & 31 \\
\hline 6 & $1 \times 10^{9}$ & 0.1 & $53.02 \pm 0.67$ & $48.64 \pm 0.22$ & 2.87 & 9.93 & 11 & 33 \\
\hline 7 & $5 \times 10^{8}$ & 0.17 & $48.58 \pm 0.19$ & $23.01 \pm 0.01$ & 4.25 & 10.82 & 16 & 36 \\
\hline 8 & $5 \times 10^{9}$ & 0.17 & $97.38 \pm 0.61$ & $74.89 \pm 0.29$ & 2.12 & 5.40 & 9 & 19 \\
\hline 9 & $1 \times 10^{10}$ & 0.17 & $119.8 \pm 0.20$ & $105.6 \pm 0.33$ & 1.69 & 4.24 & 6 & 16 \\
\hline
\end{tabular}

Table 1. DNS runs at different Rayleigh numbers $(R a)$ and Prandtl numbers $(P r)$. The parameters used in the DNS runs include the number $\left(N_{B L}\right)_{v}$ of grid points used to resolve the viscous boundary layer of dimensionless thickness $\delta_{v} / H$ and the number $\left(N_{B L}\right)_{T}$ of grid points used to resolve the thermal boundary layer of dimensionless thickness $\delta_{T} / H$. Also included are the numerically calculated values of the local Nusselt number $N u$, the Reynolds number $R e$, the normalised viscous boundary-layer thickness $\delta_{v} / H$, and the normalised thermal boundary-layer thickness $\delta_{T} / H$. For all the DNS runs, we used the same aspect ratio $\Gamma=0.2$, the same number of spectral elements across the circular cross-sectional area $\left(N_{e}\right)_{x, z}=8064$, the same number of spectral elements along the thickness direction $\left(N_{e}\right)_{y}=12$, and the same total number of spectral elements, $N_{e}=\left(N_{e}\right)_{x, z}\left(N_{e}\right)_{y}=96768$.
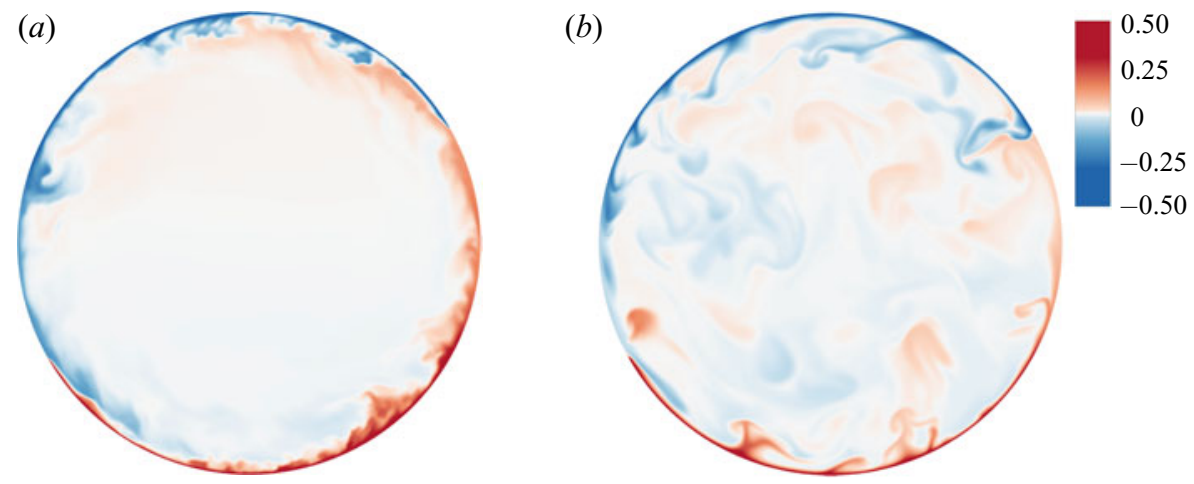

Figure 2. Contour plots of the instantaneous temperature field across the middle cross-section of the cell at ( $a) \operatorname{Pr}=0.17$ and $(b) \operatorname{Pr}=4$.4. The simulations are conducted at $R a=1 \times 10^{9}$ in a vertical thin disk with $\Gamma=0.2$. The colour code is shown with dark red for the highest dimensionless temperature 0.5 (bottom heating plate) and dark blue for the lowest dimensionless temperature -0.5 (top cooling plate). The data are shown in a linear scale ranging from -0.5 to 0.5 .

circular shape, which fits nicely to the circular sidewall. This pressure field provides a long-range homogeneous shear flow along the conducting plates. Owing to the rotational symmetry of the LSC, the pressure gradient along the conducting plates is negligibly small. The thin disk cell thus offers a simple flow structure for the study of the intrinsic properties of the LSC and boundary layers at low $\mathrm{Pr}$. The mean flow field in the high- $\mathrm{Pr}$ fluid, however, has more spatial variations and spreads deep inside the bulk region. The pressure contour in the bulk region shows a slightly tilted elliptical shape, which is caused by the accumulation of rising warm plumes on the lower right region and falling cold plumes on the upper left region. On the other hand, the mean velocity and pressure fields in convection cells of other shapes, such as thin square cells and cylinders of aspect ratio unity, are very different from those shown in figure 3. Previous numerical studies 


\section{W. Xu and others}
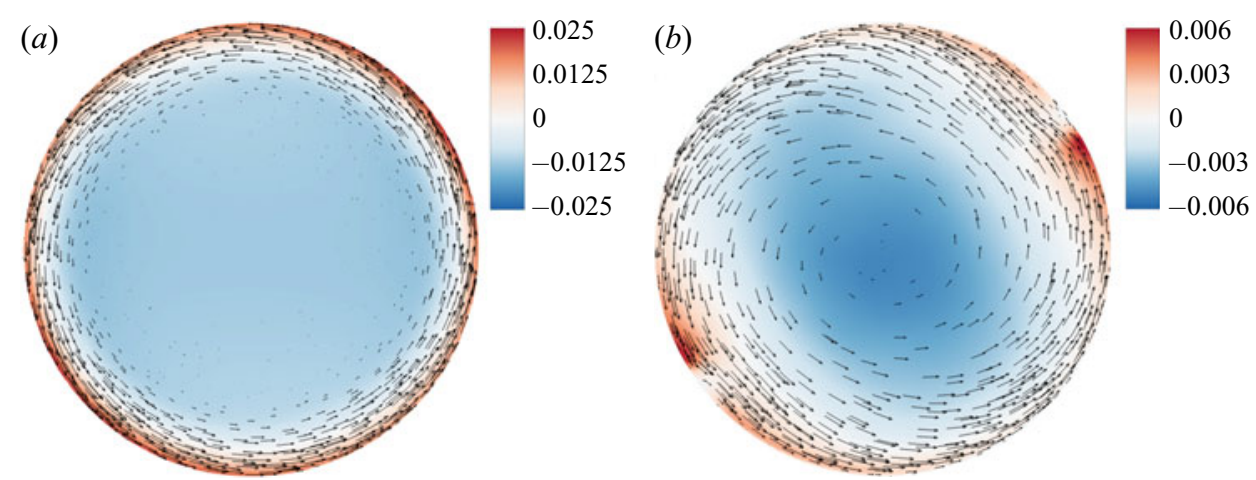

Figure 3. Vector plots of the in-plane mean velocity field and contour plots of the mean pressure field across the middle cross-section of the cell at $(a) \operatorname{Pr}=0.17$ and $(b) \operatorname{Pr}=4.4$. The simulations are conducted at $R a=$ $1 \times 10^{9}$ in a vertical thin disk with $\Gamma=0.2$. The colour code is shown with dark red for the highest pressure and dark blue for the lowest pressure in unit of $\rho U_{f}^{2}$.

(Xu 2014; Chong et al. 2018) have revealed that the mean velocity field in thin square cells has a large tilted LSC in the bulk region, which coexists with two small rolls of opposite rotation located in two opposing corners of the cell. Similar corner flows were also observed experimentally by particle image velocimetry in cylindrical cells (Sun et al. 2005). The interaction between the corner flow and LSC generates a complex structure near the conducting plates, which is different from the simple shear flow assumed by most boundary-layer theories. As shown in figure 3, no corner flow is observed in the thin disk cell.

To describe the velocity field more quantitatively, we show, in figure 4 , the normalised mean horizontal velocity profile $u(z) / U_{f}$ as a function of the normalised vertical distance $z / H$ for five different values of $P r$. At $P r=4.4$, the bulk flow behaves like a rigid-body rotation with a zero mean velocity at the cell centre. The mean velocity increases linearly with the radial distance away from the cell centre. Similar single-roll structures of the LSC were also observed in the upright cylindrical cells of aspect ratio unity (Du \& Tong 2000; Qiu \& Tong 2001; Xi et al. 2004; Sun et al. 2005; Song \& Tong 2010). As $\operatorname{Pr}$ decreases, the effect of the fluid viscosity decreases and the rotation speed of the LSC increases. The faster rotation of the LSC gives the thermal plumes less time to penetrate into the bulk region. As a result, $u(z) / U_{f}$ moves to the region near the circular sidewall. At $\mathrm{Pr}=$ 0.17 , the obtained $u(z) / U_{f}$ behaves like a near-wall jet with a sharp velocity peak near the bottom plate. The strong near-wall flow, in turn, produces a large entrainment effect carrying the thermal plumes in the narrow near-wall region. The strong coupling between the LSC and thermal plumes is self-organised so that a continuous LSC is maintained even when the thermal plumes have a short lifetime. The shape of the near-wall jet remains approximately the same when $\operatorname{Pr}$ is further reduced to 0.1 . This result suggests that the flow has reached a new steady-state regime at low $\mathrm{Pr}$.

In the following, we investigate the functional form of the normalised mean horizontal velocity profile $\tilde{u}(z) \equiv u(z) / U_{m}$ and the normalised mean temperature profile $\theta(z) \equiv$ $\left[T_{b}-T(z)\right] / \Delta_{b}$, where $T(z)$ is the actual mean temperature profile, $T_{b}$ is the bottom plate temperature and $\Delta_{b}$ is the temperature difference across the bottom thermal boundary layer. This study is carried out at a fixed $\operatorname{Pr}=0.17$ and a fixed aspect ratio $\Gamma=0.2$. As mentioned previously, $\operatorname{Pr}=0.17$ is chosen because the flow has reached a new steady-state 


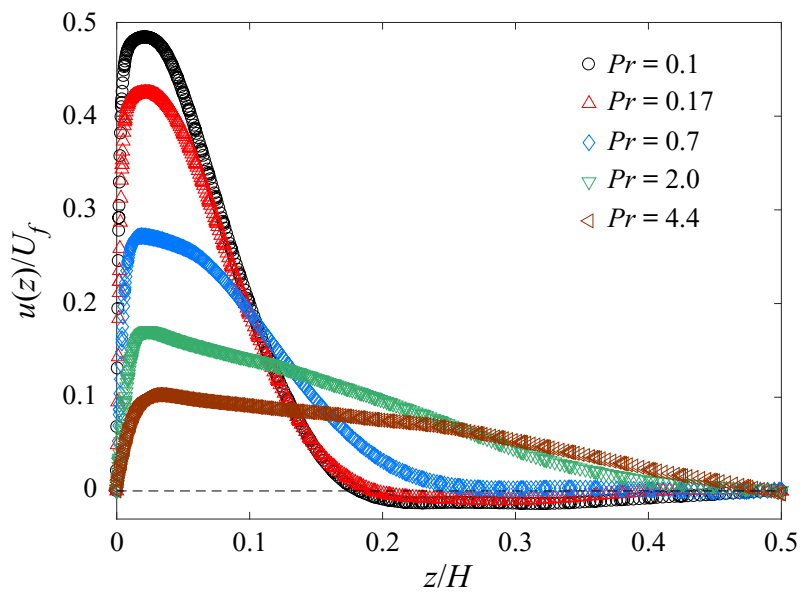

Figure 4. Normalised mean horizontal velocity profiles $u(z) / U_{f}$ as a function of the normalised vertical distance $z / H$ away from the centre of the bottom conducting plate for five values of $\operatorname{Pr}$ : 0.1 (black circles), 0.17 (red up triangles), 0.7 (blue diamonds), 2.0 (green down triangles) and 4.4 (brown left triangles). The simulations are conducted at $R a=1 \times 10^{9}$ in a vertical thin disk with $\Gamma=0.2$.

regime at low $P r$ and the DNS results can be obtained more efficiently and be used for comparison with future experiments using a gas mixture of $\mathrm{H}_{2}$ and $\mathrm{Xe}$ (Bajaj et al. 2002).

\section{Mean horizontal velocity and temperature profiles in the boundary-layer region}

To find the functional form of the mean horizontal velocity and temperature profiles in the boundary-layer region, we consider a 2-D convective flow over a long horizontal heating plate with a constant horizontal mean velocity $U_{m}$. Using the coordinate system shown in figure $1(a)$ and taking the Reynolds decomposition of the velocity field $\boldsymbol{u}(\boldsymbol{r}, t)=$ $\langle\boldsymbol{u}\rangle(\boldsymbol{r})+\boldsymbol{u}^{\prime}(\boldsymbol{r}, t)$ and temperature field $T(\boldsymbol{r}, t)=\langle T\rangle(\boldsymbol{r})+T^{\prime}(\boldsymbol{r}, t)$, we obtain the viscous and thermal boundary-layer equations

$$
\begin{gathered}
\frac{\partial u}{\partial x}+\frac{\partial w}{\partial z}=0 \\
u \frac{\partial u}{\partial x}+w \frac{\partial u}{\partial z}=-\frac{1}{\rho} \frac{\partial p}{\partial x}+v \frac{\partial^{2} u}{\partial z^{2}}-\frac{\partial\left\langle w^{\prime} u^{\prime}\right\rangle}{\partial z}, \\
u \frac{\partial T}{\partial x}+w \frac{\partial T}{\partial z}=\kappa \frac{\partial^{2} T}{\partial z^{2}}-\frac{\partial\left\langle w^{\prime} T^{\prime}\right\rangle}{\partial z}
\end{gathered}
$$

where all the time-average notation $\langle\cdot\rangle$ on the first-order terms are omitted for conciseness. These equations are obtained by applying the boundary-layer approximations $|w| \ll|u|$, $|\partial / \partial x| \ll|\partial / \partial z|$ and $\left|\partial^{2} / \partial x^{2}\right| \ll\left|\partial^{2} / \partial z^{2}\right|$, and a long time average to the dimensional form of (2.2) and (2.3). Based on the boundary-layer approximations and dimensional analysis, we find that the convection and diffusion terms in the vertical direction are much smaller than those in the horizontal direction, as shown in (4.2). As a result, only the pressure gradient and buoyancy terms remain in the viscous boundary-layer equation in the vertical direction, namely, $\rho^{-1} \partial_{z} p=g \alpha\left(T-T_{0}\right)$ (Ching et al. 2019). As shown in figure 3(a) and more quantitatively in figure 5(a), the pressure gradient term $\rho^{-1} \partial_{x} p$ in (4.2) is negligibly small, so that the vertical pressure-buoyancy balance equation does not 


\section{W. Xu and others}

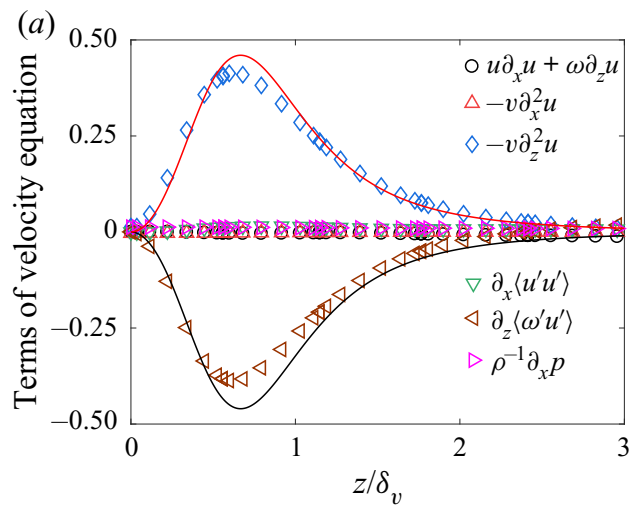

(b)

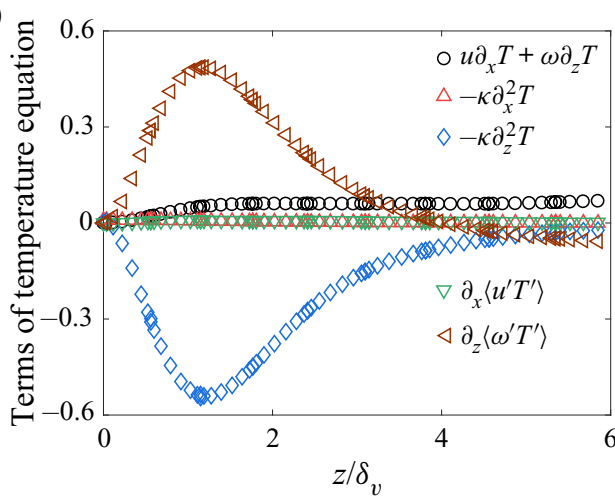

Figure 5. (a) Contributions of the mean convection term $u \partial_{x} u+w \partial_{z} u$, molecular momentum diffusion components $-v \partial_{x}^{2} u$ and $-v \partial_{z}^{2} u$, Reynolds stress gradient components $\partial_{x}\left\langle u^{\prime} u^{\prime}\right\rangle$ and $\partial_{z}\left\langle w^{\prime} u^{\prime}\right\rangle$, and pressure gradient $1 / \rho \partial_{x} p$, as a function of the normalised vertical distance $z / \delta_{v}$. The unit of the terms is $U_{f}^{2} / H$. The two solid lines show the calculated terms of the velocity equation using the analytical solution $\tilde{u}(\xi)$ in (4.14) with $a=1.19$. (b) Contributions of the mean convection term $u \partial_{x} T+w \partial_{z} T$, molecular thermal diffusion components $-\kappa \partial_{x}^{2} T$ and $-\kappa \partial_{z}^{2} T$, and turbulent heat flux gradient components $\partial_{x}\left\langle u^{\prime} T^{\prime}\right\rangle$ and $\partial_{z}\left\langle w^{\prime} T^{\prime}\right\rangle$, as a function of $z / \delta_{v}$. The unit of the terms is $U_{f} \Delta T / H$. The DNS data used for the calculations shown in $(a)$ and (b) are obtained at $\operatorname{Pr}=0.17$ and $R a=1 \times 10^{9}$ in a vertical thin disk with $\Gamma=0.2$.

affect the above viscous and thermal boundary-layer equations explicitly. For low- $\operatorname{Pr}$ RBC, we still expect the classical boundary-layer assumptions to be valid for both the viscous and thermal boundary layers. This is because the thicknesses of the two boundary layers are comparable at $\operatorname{Pr}=0.17$, with the thickness ratio between the viscous and thermal boundary layers being $\operatorname{Pr}^{1 / 2} \simeq 0.4$.

With the DNS data, we numerically calculate the contributions of each term in the mean velocity equation and the mean temperature equation. Figures $5(a)$ and $5(b)$ show, respectively, the terms of the velocity and temperature equations as a function of the normalised vertical distance $z / \delta_{v}$ away from the bottom conducting plate. Here the viscous boundary-layer thickness $\delta_{v}$ is defined as a vertical distance, at which the tangent of the mean velocity profile at the bottom plate $(z=0)$ intersects the maximum velocity $U_{m}$, namely

$$
\delta_{v}=U_{m}\left|\frac{\partial u}{\partial z}\right|_{z=0}^{-1} .
$$

It can be seen from figure 5(a) that the boundary-layer approximations hold for the mean velocity equations in the near-wall region. We verify numerically that the horizontal pressure gradient $\rho^{-1} \partial_{x} p$ is negligible at low $\operatorname{Pr}$ so that the viscous boundary-layer equation (4.2) is decoupled from the thermal boundary-layer equation (4.3) (Ching et al. 2019). In addition, we find numerically that the mean convection term, $u \partial_{x} u+w \partial_{z} u$, in (4.2) is negligibly small for this low- $\operatorname{Pr} \mathrm{RBC}$ system. As a result, only two dominant terms remain in the mean velocity equation (4.2), namely, the molecular momentum diffusion $v \partial_{z}^{2} u$ and turbulent momentum diffusion $\partial_{z}\left\langle w^{\prime} u^{\prime}\right\rangle$, which balance each other in the near-wall region,

$$
v \frac{\partial^{2} u}{\partial z^{2}}=\frac{\partial\left\langle w^{\prime} u^{\prime}\right\rangle}{\partial z} .
$$


Similarly, the mean temperature equation (4.3) also has two dominant terms, namely, the molecular thermal diffusion $\kappa \partial_{z}^{2} T$ and turbulent thermal diffusion $\partial_{z}\left\langle w^{\prime} T^{\prime}\right\rangle$. There is a small contribution from the mean convection term, $u \partial_{x} T+w \partial_{z} T$, for $R a=5 \times 10^{8}$. From the DNS results, we find the contribution of the mean convection decreases with increasing $R a$. As shown in figure $5(b)$, the mean convection can be approximately ignored for $R a=1 \times 10^{9}$. Therefore, in the $R a$ range $1 \times 10^{9} \leq R a \leq 1 \times 10^{10}$, we have

$$
\kappa \frac{\partial^{2} T}{\partial z^{2}} \simeq \frac{\partial\left\langle w^{\prime} T^{\prime}\right\rangle}{\partial z}
$$

\subsection{Mean horizontal velocity profile in the boundary-layer region}

With the turbulent viscosity $v_{t}(z)$ defined as

$$
\left\langle w^{\prime} u^{\prime}\right\rangle=-v_{t}(z) \frac{\mathrm{d} u}{\mathrm{~d} z}
$$

equation (4.5) becomes an ordinary differential equation

$$
\left(1+\frac{v_{t}}{v}\right) \frac{\mathrm{d}^{2} u}{\mathrm{~d} z^{2}}+\frac{\mathrm{d}}{\mathrm{d} z}\left(1+\frac{v_{t}}{v}\right) \frac{\mathrm{d} u}{\mathrm{~d} z}=0 .
$$

Integrating both sides and applying the definition (4.4) as the boundary condition, we obtain

$$
\frac{\mathrm{d} u}{\mathrm{~d} z}=\frac{U_{m} / \delta_{v}}{1+v_{t} / v} .
$$

The formal solution of (4.9) is given by

$$
\tilde{u}(\xi)=\int_{0}^{\xi} \frac{1}{1+v_{t}(s) / v} \mathrm{~d} s,
$$

where $\tilde{u}=u / U_{m}$ and $\xi=z / \delta_{v}$.

With the no-slip boundary condition at the bottom conducting plate, we find the Reynolds stress $\left\langle w^{\prime} u^{\prime}\right\rangle$ and its derivatives satisfy the following boundary conditions at $z=0$,

$$
\left\langle w^{\prime} u^{\prime}\right\rangle=\partial_{z}\left\langle w^{\prime} u^{\prime}\right\rangle=\partial_{z}^{2}\left\langle w^{\prime} u^{\prime}\right\rangle=0 .
$$

From the definition of $v_{t}$ in (4.7) and the linear velocity profile at the wall, i.e. $\mathrm{d} u / \mathrm{d} z \propto z^{0}$, we obtain the boundary conditions for $v_{t}$ at $\xi=0$,

$$
v_{t}(0)=\left(v_{t}\right) \xi(0)=\left(v_{t}\right)_{\xi \xi}(0)=0 .
$$

Therefore, the leading order of $v_{t}$ has the form

$$
v_{t} / v \simeq a^{3} \xi^{3}
$$

where $a$ is a constant. A similar boundary condition was also obtained for $\kappa_{t} / \kappa$ in the thermal boundary-layer equation (Shishkina et al. 2015). Substituting (4.13) into (4.10), we obtain the analytical form of the normalised horizontal velocity profile

$$
\tilde{u}(\xi)=\frac{1}{6 a} \ln \frac{(1+a \xi)^{3}}{1+(a \xi)^{3}}+\frac{\sqrt{3}}{3 a} \arctan \frac{2 a \xi-1}{\sqrt{3}}+\frac{\sqrt{3} \pi}{18 a}
$$




\section{W. Xu and others}
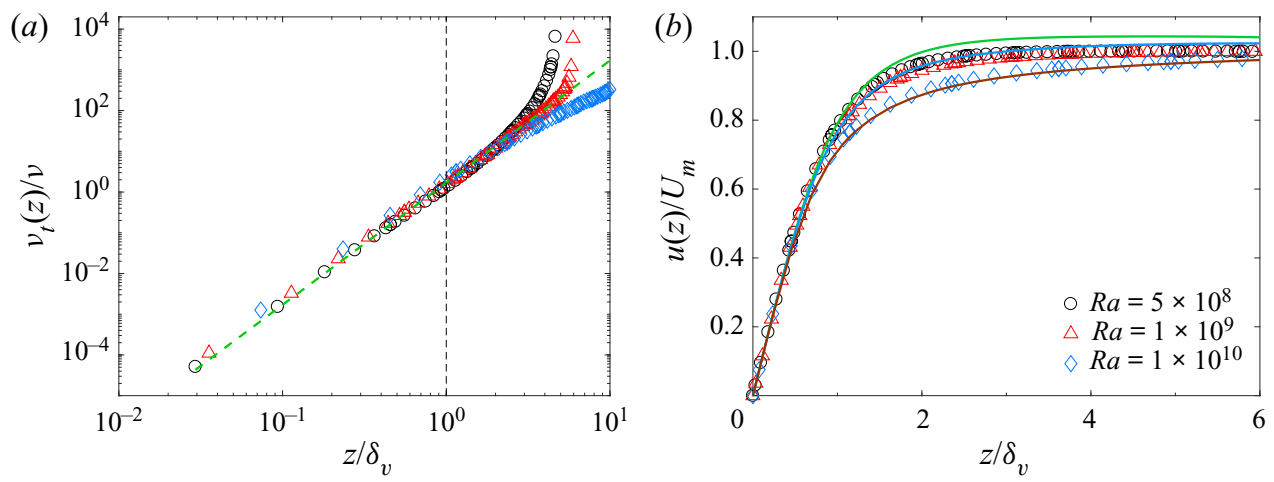

Figure 6. (a) Log-log plots of the normalised turbulent viscosity $v_{t} / v$ as a function of $z / \delta_{v}$ for $R a=5 \times 10^{8}$ (black circles), $1 \times 10^{9}$ (red triangles) and $1 \times 10^{10}$ (blue diamonds). The green dashed line shows a fit to (4.13) with $a=1.19$. The vertical dashed line indicates the edge of the viscous boundary layer with $z / \delta_{v}=1$. (b) Normalised mean horizontal velocity profile $u / U_{m}$ as a function of $z / \delta_{v}$ for $R a=5 \times 10^{8}$ (black circles), $1 \times 10^{9}$ (red triangles) and $1 \times 10^{10}$ (blue diamonds). The green, blue and brown solid lines show, respectively, the numerical solutions of (4.10) using the numerically calculated $v_{t} / v$ shown in $(a)$ for $R a=5 \times 10^{8}, 1 \times 10^{9}$ and $1 \times 10^{10}$. The DNS data used for the calculations shown in $(a)$ and $(b)$ are obtained at $\operatorname{Pr}=0.17$ in a vertical thin disk with $\Gamma=0.2$.

Figure $6(a)$ shows the normalised turbulent viscosity $v_{t} / v$ as a function of the normalised vertical distance $z / \delta_{v}$. Using (4.7), we numerically calculate $v_{t}=$ $-\left\langle w^{\prime} u^{\prime}\right\rangle /(\mathrm{d} u / \mathrm{d} z)$. For all three values of $R a$, the obtained $v_{t} / v$ is well described by (4.13) with $a=1.19$ (green dashed line) for small values of $z$ up to $z / \delta_{v} \lesssim 2$. Deviations from the green dashed line are observed when $z / \delta_{v} \gtrsim 2$. These deviations exhibit an interesting dependence on $R a$, with the obtained $v_{t} / v$ at $R a=1 \times 10^{9}$ as an exceptional case, which has a wider power-law range up to $z / \delta_{v} \simeq 6$. With the obtained $v_{t} / v$ in figure $6(a)$, we numerically compute the solution of (4.10), as shown by the three solid lines in figure $6(b)$ for the three different values of $R a$. It is seen that the agreement between the numerical solutions of (4.10) and the DNS data (open symbols) improves significantly with increasing $R a$. A small deviation between the green solid line and black circles is observed at larger values of $z / \delta_{v}$ for $R a=5 \times 10^{8}$. This is caused by the fact that for small values of $R a$, a small contribution from the mean convection term becomes noticeable, which affects the accuracy of (4.5). Because the obtained $v_{t} / v$ at $R a=1 \times 10^{9}$ is well described by (4.13) with $a=1.19$ over a wider range of $z / \delta_{v}$, the corresponding velocity profile $u / U_{m}$ as a function of $z / \delta_{v}$ (red triangles in figure $6 b$ ) is equally well described by (4.14) with the same fitting parameter $a=1.19$. By assuming (4.13) holds for distances far enough from the wall, one may use the asymptotic boundary condition, $\tilde{u}(\infty)=1$, to determine the value of $a=2 \sqrt{3} \pi / 9 \simeq 1.2$. The fitted value of $a=1.19$ is very close to this limiting value. Figure 6 thus confirms that the viscous boundary-layer model discussed earlier captures the essential physics.

\subsection{Mean temperature profile in the boundary-layer region}

Using the turbulent thermal diffusivity $\kappa_{t}(z)$ defined as

$$
\left\langle w^{\prime} T^{\prime}\right\rangle=-\kappa_{t}(z) \frac{\mathrm{d} T}{\mathrm{~d} z}
$$


equation (4.6) becomes an ordinary differential equation

$$
\left(1+\frac{\kappa_{t}}{\kappa}\right) \frac{\mathrm{d}^{2} T}{\mathrm{~d} z^{2}}+\frac{\mathrm{d}}{\mathrm{d} z}\left(1+\frac{\kappa_{t}}{\kappa}\right) \frac{\mathrm{d} T}{\mathrm{~d} z}=0 .
$$

Integrating both sides and applying the relation at the boundary,

$$
\delta_{T}=\Delta_{b}\left|\frac{\partial T}{\partial z}\right|_{z=0}^{-1},
$$

which defines the thermal boundary-layer thickness $\delta_{T}$, we obtain

$$
\frac{\mathrm{d} T}{\mathrm{~d} z}=-\frac{\Delta_{b} / \delta_{T}}{1+\kappa_{t} / \kappa} .
$$

The formal solution of (4.18) is given by

$$
\theta(\xi)=\frac{\delta_{v}}{\delta_{T}} \int_{0}^{\xi} \frac{1}{1+\kappa_{t}(s) / \kappa} \mathrm{d} s,
$$

where $\theta(z)=\left[T_{b}-T(z)\right] / \Delta_{b}$ and $\xi=z / \delta_{v}$.

For high- $\operatorname{Pr}$ RBC, the thermal boundary layer is nested within the viscous boundary layer, so that $\kappa_{t}(z)$ obeys a cubic power law similar to (4.13) across the entire thermal boundary layer. In this case, the mean temperature profile $\theta(\xi)$ takes the same functional form as shown in (4.14). This was shown previously by Shishkina et al. (2015). For low- $\mathrm{Pr}$ $\mathrm{RBC}$, however, the thermal boundary layer is thicker than the viscous boundary layer, so that the LSC may produce a strong shear on the outer portion of the thermal boundary layer, as illustrated in figure 7(a). The numerically calculated $\kappa_{t}(z)$ for $\operatorname{Pr}=0.17$ is found to obey the cubic power law in (4.13) only up to $z / \delta_{v} \simeq 1$. This is shown in figure $7(b)$. Outside the viscous boundary layer (but still within the thermal boundary layer), the obtained $\kappa_{t}(z)$ goes as $z^{2.2}$ with the power-law exponent being smaller than 3 . In this case, no analytical solution of (4.19) is available and one needs to numerically integrate (4.19). The situation is somewhat similar to a boundary layer near a partial-slip wall, as illustrated in figure $7(a)$. Owing to the large streamwise velocity at the edge of the viscous boundary layer, $\partial_{z}^{2}\left\langle w^{\prime} T^{\prime}\right\rangle$ is no longer guaranteed to be zero, so that the leading order of $\kappa_{t}$ is reduced from $z^{3}$ to $z^{2}$ (authors' unpublished observations).

With the obtained $\kappa_{t} / \kappa$ in figure 7(b), we numerically compute the solution of (4.19), as shown by the three solid lines in figure 8 for the three different values of $R a$. It is seen that the agreement between the numerical solutions of (4.19) and the DNS data (open symbols) improves significantly with increasing $R a$. Small deviations are observed at larger values of $z / \delta_{v}$ for the two lower values of $R a$. This is caused by the fact that for small values of $R a$, a small contribution from the mean convection term becomes noticeable (see figure $5 b$ ), which affects the accuracy of (4.6). The good agreement between the calculated solution of (4.19) and the DNS result at $R a=1 \times 10^{10}$ further confirms that the thermal boundary-layer model discussed previously captures the essential physics.

\section{Mean horizontal velocity profile in the bulk region}

Figure $9(a)$ shows the terms of the velocity equation as a function of the normalised vertical distance $z / H$. Compared with those terms in the boundary-layer region, all of the terms are small in the bulk region. Among them, the mean convection and the vertical 


\section{W. Xu and others}

(a)

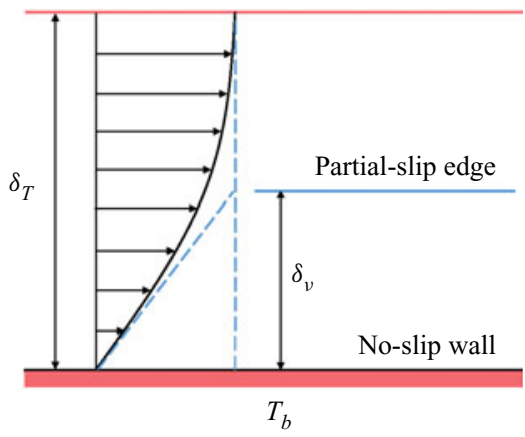

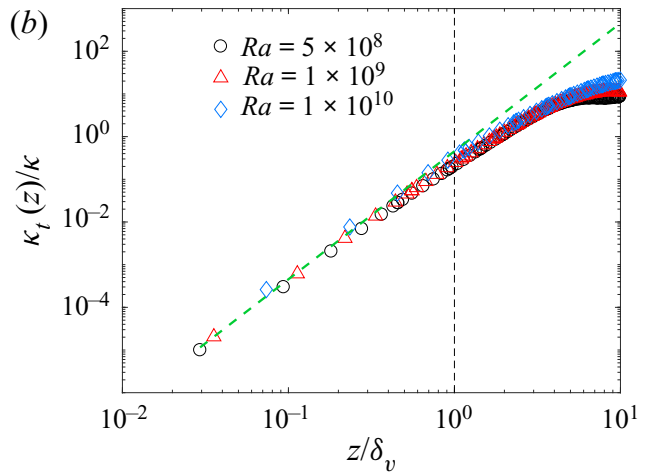

Figure 7. (a) Sketch of the viscous boundary layer and thermal boundary layer at low $P r$, in which the viscous boundary layer is nested within the thermal boundary layer. $(b) \log -\log$ plots of the normalised turbulent diffusivity $\kappa_{t} / \kappa$ as a function of $z / \delta_{v}$ for $R a=5 \times 10^{8}$ (black circles), $1 \times 10^{9}$ (red triangles) and $1 \times 10^{10}$ (blue diamonds). The green dashed line shows a cubic power law $\kappa_{t} / \kappa=0.45\left(z / \delta_{v}\right)^{3}$. The DNS data used for the calculations are obtained at $\operatorname{Pr}=0.17$ in a vertical thin disk with $\Gamma=0.2$.

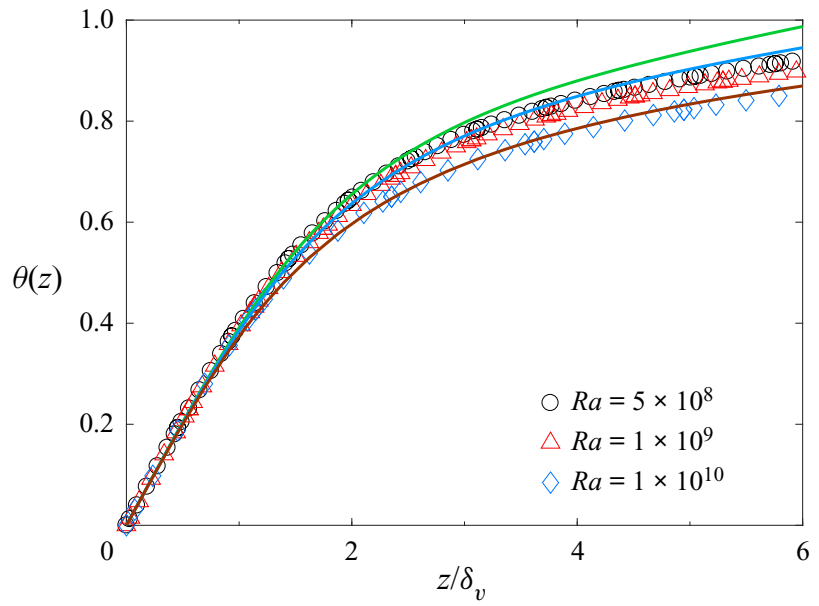

Figure 8. Normalised mean temperature profile $\theta(z)$ as a function of $z / \delta_{v}$ for $R a=5 \times 10^{8}$ (black circles), $1 \times 10^{9}$ (red triangles) and $1 \times 10^{10}$ (blue diamonds). The green, blue and brown solid lines show, respectively, the numerical solutions of (4.19) using the numerically calculated $\kappa_{t} / \kappa$ shown in figure $7(b)$ for $R a=5 \times 10^{8}$, $1 \times 10^{9}$ and $1 \times 10^{10}$. The DNS data used for the calculations are obtained at $\operatorname{Pr}=0.17$ in a vertical thin disk with $\Gamma=0.2$.

Reynolds stress gradient decay with $z / H$ much slower than the other terms. By balancing the mean convection with the vertical Reynolds stress gradient, we have

$$
u \frac{\partial u}{\partial x}+w \frac{\partial u}{\partial z}=-\frac{\partial\left\langle w^{\prime} u^{\prime}\right\rangle}{\partial z} .
$$

As shown in figure $9(b)$, the turbulent viscosity $v_{t}(x, z)$ in the bulk region is approximately independent of $z$ and, therefore, we have

$$
\left\langle w^{\prime} u^{\prime}\right\rangle=-v_{t}(x) \frac{\partial u}{\partial z} .
$$



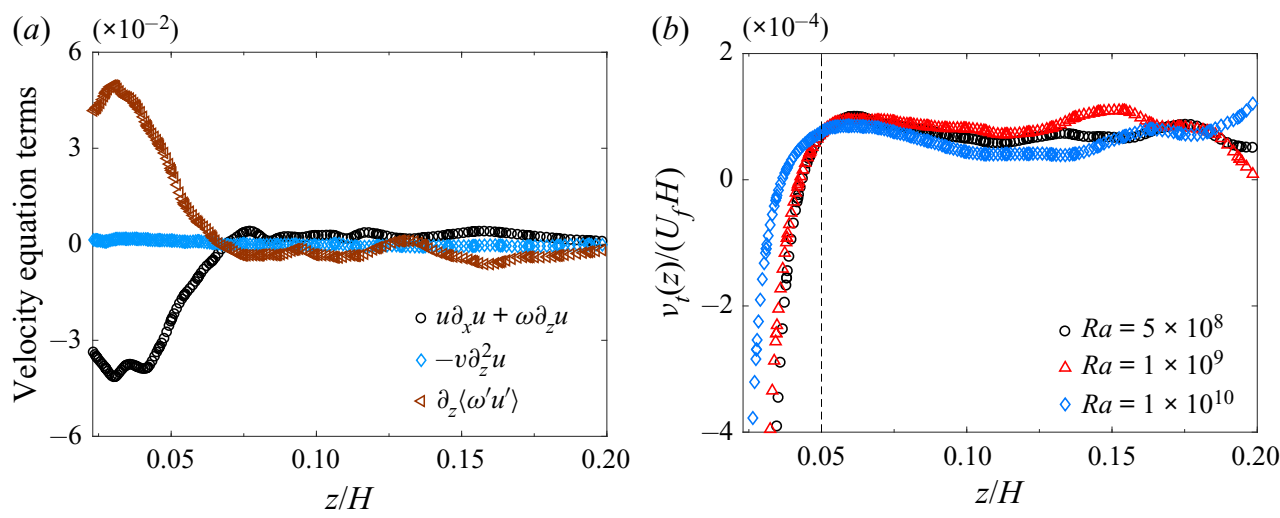

Figure 9. (a) Contributions of mean convection $u \partial_{x} u+w \partial_{z} u$ (black circles), vertical molecular momentum diffusion $-v \partial_{z}^{2} u$ (blue diamonds) and vertical Reynolds stress gradient $\partial_{z}\left\langle w^{\prime} u^{\prime}\right\rangle$ (brown triangles) as a function of the normalised vertical distance $z / H$ for $R a=1 \times 10^{9}$. The unit of the terms is $U_{f}^{2} / H$. $(b)$ Normalised turbulent viscosity $v_{t}(z) /\left(U_{f} H\right)$ as a function of $z / H$ for three values of Ra: $5 \times 10^{8}$ (black circles), $1 \times 10^{9}$ (red triangles) and $1 \times 10^{10}$ (blue diamonds). The DNS data used for the calculations shown in $(a)$ and $(b)$ are obtained at $\operatorname{Pr}=0.17$ in a vertical thin disk with $\Gamma=0.2$.

Substituting (5.2) into (5.1), we obtain

$$
u \frac{\partial u}{\partial x}+w \frac{\partial u}{\partial z}=v_{t}(x) \frac{\partial^{2} u}{\partial z^{2}} .
$$

Equation (5.3) admits a self-similarity solution for a free turbulent jet (Pope 2000). By assuming the large-scale flow in the bulk region is a half turbulent jet, we find

$$
\tilde{u}=1-\tanh ^{2}\left(\frac{z-z_{0}}{\eta}\right),
$$

where $\tilde{u}=u / U_{m}, z_{0}$ is the starting position of the half-jet, and $\eta$ represents the jet width,

$$
\eta=2 \sqrt{\frac{v_{t} x}{U_{m}}} .
$$

Figure $10(a)$ shows the normalised mean horizontal velocity profile $u(z) / U_{f}$ as a function of $z / H$. It is seen that all of the numerically calculated $u(z) / U_{f}$ for different values of $R a$ collapse approximately on to a single master curve. This suggests that the obtained $u(z) / U_{f}$ in the bulk region is approximately a scaling function of $z / H$, which is invariant with $R a$. From figures $9(b)$ and $10(a)$, we find $v_{t} \simeq 1.0 \times 10^{-4} U_{f} H$ and $U_{m} \simeq 0.42 U_{f}$. By assuming the streamwise length $x$ scales with the circumference $\pi H$ of the circular disk, i.e. $x \simeq \pi H$, we obtain the jet width $\eta \simeq 0.055 H$ from (5.5). This result thus explains why the numerically calculated $u(z) / U_{f}$ in the bulk region is a scaling function of $z / H$. In addition, figure $10(b)$ shows that the obtained $u(z) / U_{f}$ is adequately described by (5.4) and the fitted values of $\eta$ is $0.08 H$, which also agrees with the estimated value of $\eta \simeq 0.055 H$ by (5.5). The good agreement between the DNS results and the analytical solution confirms that the turbulent jet model discussed above captures the essential physics. We also compute $u(z) / U_{f}$ in the $\Gamma=0.1$ disk cell and the resulting $u(z) / U_{f}$ exhibits a similar shape of near-wall jet, as shown in figure $10(a)$. However, both the maximal value of the jet and its width show a weak $R a$-dependence. Because the jet width in the $\Gamma=0.1$ cell 

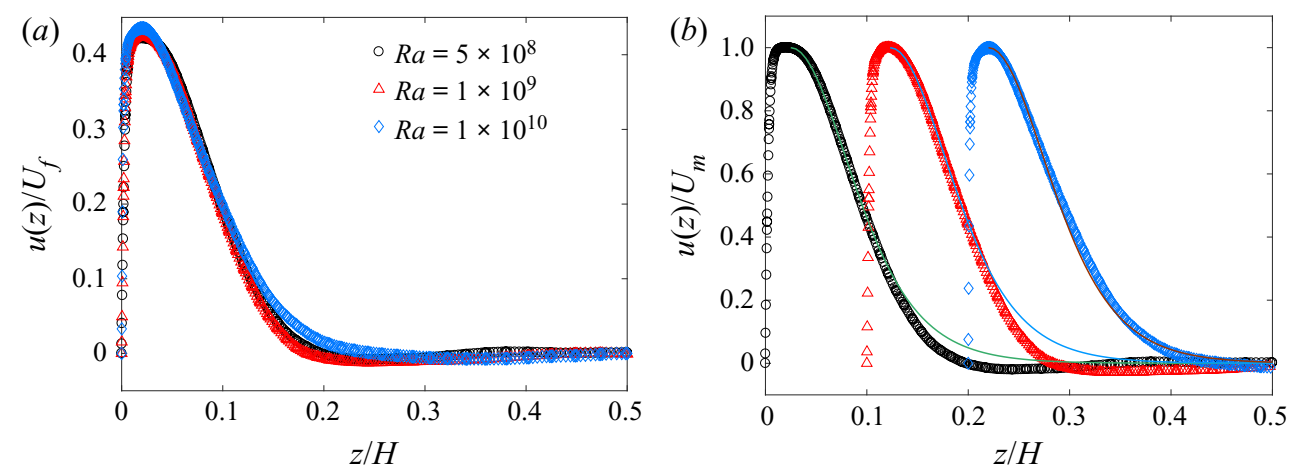

Figure 10. (a) Normalised mean horizontal velocity profiles $u(z) / U_{f}$ as a function of the normalised vertical distance $z / H$ for three different values of $R a: 5 \times 10^{8}$ (black circles), $1 \times 10^{9}$ (red triangles) and $1 \times 10^{10}$ (blue diamonds). (b) Replot of the mean horizontal velocity profiles $u / U_{m}$ normalised by its maximum value $U_{m}$ as a function of $z / H$ for $R a=5 \times 10^{8}$ (black circles), $1 \times 10^{9}$ (red triangles) and $1 \times 10^{10}$ (blue diamonds). For clarity, the origin of the red and blue curves is shift to the right by 0.1 and 0.2 normalised distances, respectively. The green, blue and brown solid lines show the same plot of (5.4) with $\eta=0.08 H$ and $z_{0}=0.02 H$. The DNS data used for the calculations shown in $(a)$ and $(b)$ are obtained at $\operatorname{Pr}=0.17$ in a vertical thin disk with $\Gamma=0.2$.

is comparable to the disk thickness, we suspect that this weak $R a$-dependence is caused by the confinement effect of the thin disk. Indeed, when the disk thickness is increased to $\Gamma=0.2$, the obtained $u(z) / U_{f}$ is found to have an approximate universal form, as shown in figure 10(a), which does not change significantly with $R a$.

\section{Summary}

We have carried out a systematic numerical study of the mean velocity and temperature profiles in turbulent Rayleigh-Bénard convection at low Prandtl numbers. The DNS runs were conducted in a vertical thin disk with the Prandtl number $(P r)$ varied in the range $0.17 \leq \operatorname{Pr} \leq 4.4$ and the Rayleigh number $(R a)$ varied between $5 \times 10^{8}$ and $1 \times 10^{10}$. For a fixed value of $R a=5 \times 10^{9}$ and with varying values of $\operatorname{Pr}$ from 4.4 to 0.17 , we find a sharp change of flow patterns for the LSC. For high- $P r$ RBC, the lifetime of thermal plumes is longer so that they have a better chance to enter the bulk region and drive the flow globally. As a result, the LSC in high- $P r$ RBC rotates like a rigid body. For low- $P r$ $\mathrm{RBC}$, however, the thermal plumes have a shorter lifetime so that they have less chance of surviving in the bulk region. Consequently, the thermal plumes are concentrated in the near-wall region and the LSC in low- $\operatorname{Pr}$ RBC behaves like a near-wall turbulent jet.

For low- $\operatorname{Pr} \mathrm{RBC}$, the viscous boundary layer is nested inside the thermal boundary layer. Thus, the strong shearing effect by the viscous boundary layer causes the thermal boundary layer to be separated into two sub-layers. This effect makes the thermal boundary layer at low $\operatorname{Pr}$ more complicated than that at high $P r$. We numerically examine the mean velocity and temperature equations in the boundary-layer region and find that both the mean horizontal velocity profile $u(z)$ and temperature profile $T(z)$ can be determined by a balance equation between the molecular diffusion and turbulent diffusion. Furthermore, the no-slip boundary condition at the bottom conducting wall dictates that both the turbulent viscosity $v_{t}(z)$ and turbulent thermal diffusivity $\kappa_{t}(z)$ as a function of distance $z$ away from the bottom conducting wall obey a cubic power law $\left(\sim z^{3}\right)$ for small values of $z$ within the viscous boundary layer. In this case, both $u(z)$ and $T(z)$ can be solved analytically and the solutions agree well with the DNS data. Owing to the large streamwise velocity at the edge of the viscous boundary layer, the leading order of the obtained $\kappa_{t}(z)$ at 
larger values of $z$ outside the viscous boundary layer (but still within the thermal boundary layer) is reduced from $z^{3}$ to $z^{2}$. In this case, the mean temperature profile $T(z)$ can be solved numerically using the calculated $\kappa_{t}(z)$.

For a fixed value of $\operatorname{Pr}=0.17$ and with varying $R a$ in the range $5 \times 10^{8} \leq R a \leq$ $1 \times 10^{10}$, we find that the mean horizontal velocity profile $u(z)$ can be determined by an equation with the mean convection balanced by turbulent diffusion with a $z$-independent turbulent viscosity $v_{t}$. This balance equation admits a self-similarity jet solution, which fits the DNS data well. The width $\eta$ of the near-wall jet is determined by the diffusion equation $\eta^{2}=4 v_{t} \tau$, where $\tau \simeq \pi H / U_{m}$ is the turnover time of the LSC with $\pi H$ being the circumference of the circular disk and $U_{m}$ is the maximum velocity of the LSC. Our DNS data also reveal that the azimuthal velocity profiles at different polar angles $\theta$ have a similar near-wall jet shape, as shown in figure 10 . However, the peak velocity $U_{m}$ has a weak angular dependence. We believe that this is caused by the angular dependence of the buoyancy term, $g \sin \theta$, where $g$ is the gravitational acceleration. Owing to this buoyancy term, the momentum equation is coupled to the thermal equation, which makes the problem difficult to solve mathematically. Here we choose the vertical $z$-direction with $\theta=0$, so that the two equations are decoupled. In this case, we find a simple mathematical solution to describe the vertical profile of the mean horizontal velocity $u(z)$, which captures the essential feature of the LSC in low- $\operatorname{Pr}$ RBC.

Our work thus provides a full characterisation of the mean velocity and temperature profiles in low- $\operatorname{Pr}$ RBC. This characterisation allows us to further understand the intricate interplay between the LSC, plume emission and boundary-layer dynamics, and pinpoint the physical mechanism for the emergence of a pronounced LSC in low- $P r$ RBC. This study lays down a foundation for further study of the effects of cell shape and confinement by cell end walls, as reported by Chong et al. (2018) for a different cell geometry.

Acknowledgements. We would like to thank Prof. Emily S. C. Ching and Dr. G. Y. Cao for useful discussions. This work was supported in part by the Hong Kong Research Grants Council under grant nos. 16301719 (P.T.), N_HKUST604/19 (P.T.) and 16305819 (X.W.) and by the National Natural Science Foundation of China under grant nos. 11772111 (X.H.), 91952101 (X.H.), 11702128 (S.H.), 11961160719 (S.H.) and 91752201 (S.H.). J.S. acknowledges support by the Deutsche Forschungsgemeinschaft within the Priority Programme DFG-SPP 1881 on Turbulent Superstructures.

Declaration of Interests. The authors report no conflict of interest.

\section{Author ORCIDs.}

(1) Wei Xu https://orcid.org/0000-0003-1174-9175;

() Yin Wang https://orcid.org/0000-0002-6572-4902;

(1) Xiaozhou He https://orcid.org/0000-0001-8116-889X;

(ㄱ) Xiaoping Wang https://orcid.org/0000-0002-8853-8926;

(ㄱ) Jörg Schumacher https://orcid.org/0000-0002-1359-4536;

(1) Shi-Di Huang https://orcid.org/0000-0001-5719-6428;

(D) Penger Tong https://orcid.org/0000-0002-6340-8084.

\section{REFERENCES}

Ahlers, G., Grossmann, S. \& Lohse, D. 2009 Heat transfer and large scale dynamics in turbulent Rayleigh-Bénard convection. Rev. Mod. Phys. 81, 503-537.

Bajaj, K.M., Ahlers, G. \& Pesch, W. 2002 Rayleigh-Bénard convection with rotation at small Prandtl numbers. Phys. Rev. E 65, 056309.

Belmonte, A., Tilgner, A. \& Libchaber, A. 1993 Boundary layer length scales in thermal turbulence. Phys. Rev. Lett. 70, 4067-4070. 


\section{W. Xu and others}

Belmonte, A., Tilgner, A. \& Libchaber, A. 1994 Temperature and velocity boundary layers in turbulent convection. Phys. Rev. E 50, 269-279.

Brodova, I.G., Popel, P.S. \& Eskin, G.I. 2001 Liquid Metal Processing: Applications to Aluminium Alloy Production. CRC Press.

Brown, E. \& Ahlers, G. 2008 Azimuthal asymmetries of the large-scale circulation in turbulent Rayleigh-Bénard convection. Phys. Fluids 20, 105105.

Brown, E. \& Ahlers, G. 2009 The origin of oscillations of the large-scale circulation of turbulent Rayleigh-Bénard convection. J. Fluid Mech. 638, 383-400.

Ching, E.S., Leung, H., Zwirner, L. \& Shishina, O. 2019 Velocity and thermal boundary layer equations for turbulent Rayleigh-Bénard convection. Phys. Rev. Res. 1, 033037.

Chong, K.L., Wagner, S., Kaczorowski, M., Shishina, O. \& XiA, K.-Q. 2018 Effect of Prandtl number on heat transport enhancement in Rayleigh-Bénard convection under geometrical confinement. Phys. Rev. Fluids 3, 013501.

Cioni, S., Ciliberto, S. \& Sommeria, J. 1997 Strongly turbulent Rayleigh-Bénard convection in mercury: comparison with results at moderate Prandtl number. J. Fluid Mech. 335, 111-140.

Deville, M.O., Fischer, P.F. \& Mund, E.H. 2002 High-order Methods for Incompressible Fluid Flow. Cambridge University Press.

Du, Y.-B. \& Tong, P. 2000 Turbulent thermal convection in a cell with ordered rough boundaries. J. Fluid Mech. 407, 57-84.

FISCHER, P.F. 1997 An overlapping Schwarz method for spectral element solution of the incompressible Navier-Stokes equations. J. Comput. Phys. 133, 84-101.

Funfschilling, D. \& Ahlers, G. 2004 Plume motion and large-scale circulation in a cylindrical Rayleigh-Bénard cell. Phys. Rev. Lett. 92, 194502.

Glatzmaier, G.A. \& Roberts, P.H. 1995 A three-dimensional self-consistent computer simulation of a geomagnetic field reversal. Nature 377, 203-209.

Grossmann, S. \& Lohse, D. 2000 Scaling in thermal convection: a unifying theory. J. Fluid Mech. 407, 27-56.

GRÖтZвACH, G. 1983 Spatial resolution requirements for direct numerical simulation of the Rayleigh-Bénard convection. J. Comput. Phys. 49, 241-264.

Hanasoge, S., Gizon, L. \& Sreenivasan, K.R. 2016 Seismic sounding of convection in the Sun. Annu. Rev. Fluid Mech. 48, 191-217.

Hartmann, D.L., Moy, L.A. \& FU, Q. 2001 Tropical convection and the energy balance at the top of the atmosphere. J. Clim. 14, 4495-4511.

HogG, J. \& Ahlers, G. 2013 Reynolds-number measurements for low-Prandtl-number turbulent convection of large-aspect-ratio samples. J. Fluid Mech. 725, 664-680.

IHLI, T., et al. 2008 Review of blanket designs for advanced fusion reactors. Fusion Engng Des. 83, 912-919.

Ji, D. \& BRown, E. 2020 Low-dimensional model of the large-scale circulation of turbulent Rayleigh-Bénard convection in a cubic container. Phys. Rev. Fluids 5, 064606.

KADANOFF, L.P. 2001 Turbulent heat flow: structures and scaling. Phys. Today 54, 34-39.

Krishnamurti, R. \& HowARD, L.N. 1981 Large-scale flow generation in turbulent convection. Proc. Natl Acad. Sci. USA 78, 1981-1985.

LAndAu, L. \& Lifshitz, E. 1987 Fluid mechanics. In Course of Theoretical Physics, 2nd English edn, vol. 6. Pergamon Press (translated from the Russian by JB Sykes and WH Reid).

LUI, S.-L. \& XIA, K.-Q. 1998 Spatial structure of the thermal boundary layer in turbulent convection. Phys. Rev. E 57, 5494-5503.

Mashiko, T., Tsuji, Y., Mizuno, T. \& Sano, M. 2004 Instantaneous measurement of velocity fields in developed thermal turbulence in mercury. Phys. Rev. E 69, 036306.

van Der Poel, E.P., Stevens, R.J. \& Lohse, D. 2013 Comparison between two-and three-dimensional Rayleigh-Bénard convection. J. Fluid Mech. 736, 177-194.

Pope, S.B. 2000 Turbulent Flows. Cambridge University Press.

Du Puits, R., Resagk, C. \& Thess, A. 2010 Measurements of the instantaneous local heat flux in turbulent Rayleigh-Bénard convection. New J. Phys. 12, 075023.

Du Puits, R., Resagk, C. \& Thess, A. 2013 Thermal boundary layers in turbulent Rayleigh-Bénard convection at aspect ratios between 1 and 9. New J. Phys. 15, 013040.

QIU, X.-L. \& Tong, P. 2001 Large-scale velocity structures in turbulent thermal convection. Phys. Rev. E 64, 036304.

VAN ReEuwijk, M., Jonker, H.J. \& HAnjalić, K. 2008 Wind and boundary layers in Rayleigh-Bénard convection. II. Boundary layer character and scaling. Phys. Rev. E 77, 036312. 


\section{Mean velocity and temperature profiles at low Prandtl numbers}

Scheel, J., Kim, E. \& White, K. 2012 Thermal and viscous boundary layers in turbulent Rayleigh-Bénard convection. J. Fluid Mech. 711, 281-305.

Scheel, J.D., Emran, M.S. \& Schumacher, J. 2013 Resolving the fine-scale structure in turbulent Rayleigh-Bénard convection. New J. Phys. 15, 113063.

SCHEel, J.D. \& SchumacheR, J. 2014 Local boundary layer scales in turbulent Rayleigh-Bénard convection. J. Fluid Mech. 758, 344-373.

Scheel, J.D. \& Schumacher, J. 2016 Global and local statistics in turbulent convection at low Prandtl numbers. J. Fluid Mech. 802, 147-173.

SCHEEL, J.D. \& SCHUMACHER, J. 2017 Predicting transition ranges to fully turbulent viscous boundary layers in low Prandtl number convection flows. Phys. Rev. Fluids 2, 123501.

Schlichting, H. \& Gersten, K. 2016 Boundary-layer Theory. Springer.

Schumacher, J., Bandaru, V., Pandey, A. \& Scheel, J.D. 2016 Transitional boundary layers in low-Prandtl-number convection. Phys. Rev. Fluids 1, 084402.

Schumacher, J., Götzfried, P. \& SCheel, J.D. 2015 Enhanced enstrophy generation for turbulent convection in low-Prandtl-number fluids. Proc. Natl Acad. Sci. USA 112, 9530-9535.

Schumacher, J. \& SReEnivasan, K.R. 2020 Colloquium: unusual dynamics of convection in the Sun. Rev. Mod. Phys. 92, 041001.

Shi, N., Emran, M.S. \& Schumacher, J. 2012 Boundary layer structure in turbulent Rayleigh-Bénard convection. J. Fluid Mech. 706, 5-33.

SHISHKINA, O., HORN, S. \& WAGNER, S. 2013 Falkner-Skan boundary layer approximation in Rayleigh-Bénard convection. J. Fluid Mech. 730, 442-463.

Shishina, O., Horn, S., Wagner, S. \& Ching, E.S. 2015 Thermal boundary layer equation for turbulent Rayleigh-Bénard convection. Phys. Rev. Lett. 114, 114302.

Shishina, O., Stevens, R.J., Grossmann, S. \& Lohse, D. 2010 Boundary layer structure in turbulent thermal convection and its consequences for the required numerical resolution. New J. Phys. 12, 075022.

Song, H., Brown, E., Hawkins, R. \& Tong, P. 2014 Dynamics of large-scale circulation of turbulent thermal convection in a horizontal cylinder. J. Fluid Mech. 740, 136-167.

SonG, H. \& ToNG, P. 2010 Scaling laws in turbulent Rayleigh-Bénard convection under different geometry. Europhys. Lett. 90, 44001.

Song, H., Villermaux, E. \& Tong, P. 2011 Coherent oscillations of turbulent Rayleigh-Bénard convection in a thin vertical disk. Phys. Rev. Lett. 106, 184504.

Stevens, R.J., Zhou, Q., Grossmann, S., Verzicco, R., Xia, K. -Q. \& Lohse, D. 2012 Thermal boundary layer profiles in turbulent Rayleigh-Bénard convection in a cylindrical sample. Phys. Rev. E 85, 027301.

Stevenson, D. 1981 Models of the Earth's core. Science 214, 611-619.

SugiYama, K., et al. 2010 Flow reversals in thermally driven turbulence. Phys. Rev. Lett. 105, 034503.

Sun, C., XIA, K.-Q. \& Tong, P. 2005 Three-dimensional flow structures and dynamics of turbulent thermal convection in a cylindrical cell. Phys. Rev. E 72, 026302.

Takeshita, T., Segawa, T., Glazier, J.A. \& SAno, M. 1996 Thermal turbulence in mercury. Phys. Rev. Lett. 76, 1465-1468.

Tsuji, Y., Mizuno, T., Mashiko, T. \& SAno, M. 2005 Mean wind in convective turbulence of mercury. Phys. Rev. Lett. 94, 034501.

Vogt, T., Horn, S., Grannan, A.M. \& Aurnou, J.M. 2018 Jump rope vortex in liquid metal convection. Proc. Natl Acad. Sci. USA 115, 12674-12679.

WAGNER, S., SHISHKINA, O. \& WAGNER, C. 2012 Boundary layers and wind in cylindrical Rayleigh-Bénard cells. J. Fluid Mech. 697, 336-366.

WANG, K., et al. 2014 Lithium-antimony-lead liquid metal battery for grid-level energy storage. Nature 514, $348-350$.

WANG, Y., HE, X. \& Tong, P. 2016 Boundary layer fluctuations and their effects on mean and variance temperature profiles in turbulent Rayleigh-Bénard convection. Phys. Rev. Fluids 1, 082301.

Wang, Y., Xu, W., He, X., Yik, H., Wang, X., Schumacher, J. \& Tong, P. 2018 Boundary layer fluctuations in turbulent Rayleigh-Bénard convection. J. Fluid Mech. 840, 408-431.

XI, H.-D., LAM, S. \& XIA, K.-Q. 2004 From laminar plumes to organized flows: the onset of large-scale circulation in turbulent thermal convection. J. Fluid Mech. 503, 47-56.

XI, H.-D., Zhou, S.-Q., Zhou, Q., ChAn, T.-S. \& XIA, K.-Q. 2009 Origin of the temperature oscillation in turbulent thermal convection. Phys. Rev. Lett. 102, 044503.

XU, W. 2014 Numerical simulation of thermal convection in three-dimensional confined rectangular cell and characteristics of flow pattern and heat transfer. MSc thesis, Sun Yat-sen University, China.

ZHOU, Q. \& XIA, K.-Q. 2010 Measured instantaneous viscous boundary layer in turbulent Rayleigh-Bénard convection. Phys. Rev. Lett. 104, 104301. 


\section{W. Xu and others}

ZHou, Q. \& XIA, K.-Q. 2013 Thermal boundary layer structure in turbulent Rayleigh-Bénard convection in a rectangular cell. J. Fluid Mech. 721, 199-224.

Zocchi, G., Moses, E. \& Libchaber, A. 1990 Coherent structures in turbulent convection, an experimentalstudy. Physica A (Amsterdam) 166, 387-407.

ZÜrner, T., SChindler, F., Vogt, T., EcKert, S. \& Schumacher, J. 2019 Combined measurement of velocity and temperature in liquid metal convection. J. Fluid Mech. 876, 1108-1128. 